Review

\title{
Current Approaches and Challenges in Monitoring Treatment Responses in Breast Cancer
} \author{
Aaron D. Kirkpatrick ${ }^{1}$, Chris Gallagher ${ }^{2}$, Aviram Nissan ${ }^{3}$, Leonard Henry ${ }^{4}$, Alexander Stojadinovic ${ }^{5}$, \\ George E. Peoples ${ }^{1}$, Nathan M. Shumway ${ }^{1}$ \\ 1. San Antonio Military Medical Center (SAMMC), 3551 Roger Brooke Dr., Ft. Sam Houston, TX 78234, USA \\ 2. Walter Reed National Military Medical Center (NMMC), 8901 Wisconsin Ave Bethesda, MD 20814, USA \\ 3. Hadassah Medical Center, Kiryat Hadassah, POB 12000, Jerusalem, 91120, Israel \\ 4. IU Health Goshen, 200 High Park Ave., Goshen, IN 46526, USA \\ 5. Bon Secours Cancer Institute, 6505 West Broad St., Richmond, VA 23230, USA
}

Lindsey J. Graham ${ }^{1}{ }^{凶}$, Matthew P. Shupe ${ }^{1}$, Erika J. Schneble ${ }^{1}$, Frederick L. Flynt ${ }^{1}$, Michael N. Clemenshaw ${ }^{1}$,

$\triangle$ Corresponding author: Lindsey J. Graham, Brooke Army Medical Center, Department of Hematology/Oncology, 3551 Roger Brooke Dr., Ft. Sam Houston, TX 78234. Office: 210-916-0705; Fax: 210-916-3366; Email: Lindsey.j.graham.mil@mail.mil

(c) Ivyspring International Publisher. This is an open-access article distributed under the terms of the Creative Commons License (http://creativecommons.org/ licenses/by-nc-nd/3.0/). Reproduction is permitted for personal, noncommercial use, provided that the article is in whole, unmodified, and properly cited.

Received: 2013.06.29; Accepted: 2013.08.0I; Published: 20I4.0I.05

\begin{abstract}
Monitoring response to treatment is a key element in the management of breast cancer that involves several different viewpoints from surgery, radiology, and medical oncology. In the adjuvant setting, appropriate surgical and pathological evaluation guides adjuvant treatment and follow up care focuses on detecting recurrent disease with the intention of improving long term survival. In the neoadjuvant setting, assessing response to chemotherapy prior to surgery to include evaluation for pathologic response can provide prognostic information to help guide follow up care. In the metastatic setting, for those undergoing treatment, it is crucial to determine responders versus non-responders in order to help guide treatment decisions. In this review, we present the current guidelines for monitoring treatment response in the adjuvant, neoadjuvant, and metastatic setting. In addition, we also discuss challenges that are faced in each setting.
\end{abstract}

Key words: Breast cancer; Treatment monitoring; Future directions; Adjuvant; Neo-adjuvant; Metastatic

\section{INTRODUCTION}

In early 2012, the number of breast cancer survivors in the United States was nearly 3 million with more than two hundred thousand additional women estimated to develop breast cancer in that same year [1]. Screening and improved adjuvant treatments have increased breast cancer survival rates since the mid-1970s, with current 5 year survival rates at nearly $90 \%$ [2]. Despite these significant improvements, the chances of recurrent or relapsed disease are sobering. Recent analysis from the ATLAS (Adjuvant Tamoxifen Longer Against Shorter) trial, showed that while women with ER-positive breast cancer had an improvement in survival with 10 years of tamoxifen therapy versus the standard 5 years of therapy, the long-term recurrence rate was still 21.4\% [3]. Despite several good options for treatment for local recurrence, metastatic relapse remains an incurable disease in most instances. Currently, the average survival for women with metastatic disease is less than 2 years. A recent meta-analysis of women with metastatic breast cancer receiving first line taxane-based chemotherapy showed a median survival of 19.3 months [4]. Perhaps earlier detection from more aggressive monitoring could lead to improved treatment strategies and possibly improved survival.

In this paper, we discuss current strategies for monitoring treatment response in various clinical settings that are encountered in everyday oncology 
practice. First, we will discuss monitoring for breast cancer recurrence after adjuvant therapy. Many women will undergo surgery, radiation, and chemotherapy in an attempt to cure their disease. The period following completion of therapy can be difficult for women. Up to a third of breast cancer survivors will experience distress and of those who experience these symptoms, close to $70 \%$ consider referral to a specialist [5]. Before another blood test or radiograph is ordered, we must consider the evidence for such monitoring and more importantly consider the psychological impact the result of that test may have on survivors. In this review, we will discuss the current guidelines for disease monitoring in the adjuvant setting. We will then explore the subset of patients who undergo neoadjuvant treatment prior to surgical resection of their disease. The role of radiology, tissue pathology and surrogate markers of response are quickly changing and will be discussed. Finally, we will elaborate on ways to monitor treatment response in the metastatic setting. We will describe challenges faced in each of these clinical settings and in the follow on review; we will explore future directions to help guide clinicians and patients who are fighting this deadly disease.

\section{MONITORING TREATMENT RESPONSE IN THE ADJUVANT SETTING}

After completion of adjuvant therapy, follow up care focuses on detecting recurrent disease with the intention of improving long term survival. Surgical aspects focus on complete pathologic assessment of disease that will guide decisions on adjuvant therapy. Radiographic studies then provide non-invasive means to detect recurrent or new disease while regular follow up with a medical oncologist to discuss any new or concerning symptoms also aims to detect recurrent disease as early as possible in an attempt to improve survival by early treatment of recurrence or metastases.

\section{SURGICAL ASPECTS}

The initial treatment of early staged breast cancer is surgical removal of the tumor. For years, women have chosen between mastectomy with or without reconstruction versus breast conserving surgery (BCS) consisting of lumpectomy followed by radiation. For invasive breast cancers or even DCIS under certain circumstances (large, grade 3, or micro-invasive disease), a sentinel lymph node biopsy (SLNB) is performed at the same time as the tumor removal. Axillary lymph node dissection (AxLND) as an initial indicator of lymph node status is rarely indicated in current practice. Standard of care (SoC) dictates that the primary cancer be completely removed requiring assessment of the surgical margins. Likewise, if a SLNB is performed, thorough pathologic assessment including serial step sectioning and immunohistochemical staining (IHC) determines the presence of metastatic cancer. The margin status and the detection and quantification of nodal disease burden dictate the adequacy of surgical treatment and the need for additional procedures to ensure optimization of surgical therapy. Nodal status, tumor size, tumor characteristics, and choice of surgery will dictate additional adjuvant therapies like chemotherapy, radiation, hormonal therapy, and immunotherapy.

\section{Margin Assessment}

Margin assessment is most straightforward after mastectomy. With complete removal of the breast, wide margins are usually obtained except for instances where the tumor lies close to the skin or chest wall. In the case of the latter, removal of the pectoralis fascia usually results in a clear deep margin. Rarely is this biologic barrier penetrated in early- staged breast cancers. More locally-advanced cancers can penetrate into the pectoralis major muscle, but even this is not deemed chest wall involvement and does not up-stage a patient. Muscle can be easily removed to clear this margin if necessary.

The superficial margin is often more problematic with the indistinct transition between subcutaneous fat and breast tissue. This has become particularly problematic with the trend toward skin-sparing mastectomies. Furthermore, the trend toward immediate reconstructions can be complicated by a positive margin on final pathology that requires re-excision risking exposure of a tissue-expander. In an attempt to avoid positive superficial margins, thin flaps are often created risking skin necrosis and loss which complicates and even delays SoC adjuvant therapies. The surgical team must carefully balance the decisions between good oncologic surgery and cosmetic outcomes. New techniques and/or technologies for assessing margins and flap viability would help alleviate some of these dilemmas.

BCS requires a margin-negative lumpectomy. The extent of the margin has been an ongoing debate for decades. Some centers consider "no tumor on ink" as adequate while others want to see a minimum of $2-3 \mathrm{~mm}$ of negative margin surrounding the tumor. Regardless of the extent, the margins should be free of tumor to ensure low local recurrence rates [6]. The assessment of these margins has been another area of ongoing interest. The surgeon balances the size and adequacy of the lumpectomy with cosmesis. A positive/close margin requires another surgical procedure which delays ongoing care, causes the patient 
additional stress, and has a substantial added cost to breast cancer care. Better ways to assess the margins in real-time could limit surgery, improve cosmesis, and avoid second procedures.

\section{Nodal Assessment}

SLNB has become the SoC preferred method for staging the axilla. The precise technique varies somewhat from center to center as to the use of radiotracer with or without blue dye, timing, and location of injections. Regardless, the thorough examination of the identified SLN can reveal the presence or absence of metastatic disease, but more importantly, it can reveal the extent of disease. Isolated tumor cells $(<0.2 \mathrm{~mm})$ are noted but are not considered as N1 disease. Micro-metastases $(0.2-2 \mathrm{~mm})$ are staged as N1mic, and in the presence of a T1 tumor remains stage $1 \mathrm{~b}$ disease. Macro-metastases $(>2 \mathrm{~mm})$ are staged as N1-3 based on number and location of the positive nodes (AJCC Staging Manual, $7^{\text {th }}$ edition). Based on recently published data and NCCN guidelines, the previous SoC requirement for AxLND for any N1 disease (including N1mic) is changing [7]. Currently, patients that meet the Z-11 criteria and are receiving BCS, may forego AxLND. This obviously begs the question as to whether other patients undergoing mastectomies and with low nodal disease burdens really benefit from AxLND.

Since the current SoC is follow-on AxLND for node-positive disease after SLN except in the fairly narrow Z-11 group, there has been considerable interest in determining the presence or absence of metastatic disease at the time of SLNB in order to proceed with AxLND during the same anesthetic and procedure. This would obviously obviate the need for unnecessary waiting, stress, and second procedures for the patients requiring full axillary staging. Touch preps, frozen sections, and real-time PCR-based methodologies have been proposed and deployed with variable results. Rapid and accurate technologies could offer a significant advantage for those patients that truly need an AxLND.

\section{RADIOLOGY ASPECTS}

Improved screening and treatment strategies for breast cancer have contributed to a significant decrease in breast cancer-related mortality over the past 20 to 30 years. BCS with radiation results in similar survival outcomes as mastectomy with local recurrence in the ipsilateral breast occurring 6-9\% at 5 years and $14-20 \%$ at 20 years [8]. Early detection of asymptomatic local recurrence via appropriate surveillance techniques, to include breast imaging, improves long-term survival when compared to late symptomatic detection [9-11]. Therefore sensitive, non-invasive, and cost-effective surveillance strategies to detect early local recurrence are necessary.

\section{Mammography}

Mammography is the mainstay of surveillance imaging following curative treatment of breast cancer with $8 \%-50 \%$ of ipsilateral recurrence and $18 \%-80 \%$ of contralateral metachronous cancer detected by mammography alone [12]. Most treatment guidelines including the American Society for Clinical Oncology and the NCCN suggest annual mammography following breast conservation therapy [13, 14]. This recommendation is based upon expert opinion, as there are no adequate randomized controlled trials demonstrating mammography's benefit in the setting of surveillance.

Most guidelines suggest patients obtain their the first post-treatment mammogram " 1 year after the initial mammogram that leads to diagnosis but no earlier than 6 months after definitive radiation therapy"[14]. Although researchers agree that regular surveillance mammography in women diagnosed with early stage breast cancer improves long-term outcomes, the optimal interval for mammographic follow-up is currently debated. Some studies suggest benefit from biannual mammography for 2-5 years following treatment, while other studies and most major treatment guidelines (including ASCO and NCCN) support annual mammography following breast conservation therapy $[15,16]$. One retrospective study as well as a meta-analysis of surveillance mammography found no benefit to 6-month interval screening mammography while a recent retrospective single institution review suggested benefit from 5 years of biannual mammographic surveillance [12, 17-19].

There are no randomized clinical trials evaluating the effectiveness of breast magnetic resonance imaging (MRI), ultrasound, or positron-emission computed tomography (PET/CT) in the setting of breast cancer surveillance. The majority of literature regarding each modality is retrospective in nature. Thus, there is no definitive evidence to support any of these modalities as a primary imaging modality for surveillance [20]. Identifying the optimal imaging modality for surveillance imaging remains a significant challenge. Currently there is no ideal single modality for imaging surveillance that is non-invasive, cost effective, and has the appropriate balance of sensitivity and specificity. Although mammography is able to detect $25-45 \%$ of recurrences, the post-operative and post-radiation changes of breast conservation therapy decrease mammography's sensitivity and specificity compared with a standard screening population [21]. Thus, the role of other breast 
imaging modalities as an adjunct screening tool to mammography and clinical exam is an active area of investigation.

\section{Breast MRI}

Breast MRI demonstrates high sensitivity and specificity for the detection of local recurrence in multiple studies [22-24]. It also has high sensitivity, specificity, and accuracy in differentiating post-operative scar from recurrent tumor [25]. While breast MRI is superior to other modalities as a single option, it is expensive, resource intensive, and less tolerable than mammography or ultrasound $[25,26]$. There is wide variability in the use of breast MRI for follow-up of women after breast conservation therapy. The American College of Radiology practice guidelines for breast MR state that MRI is useful in women with a history of breast cancer and suspicion of recurrence when clinical, mammographic, or sonographic findings are inconclusive [20]. Similarly, an American Cancer Society panel concluded that the increased risk of local recurrence or contralateral metachronous disease due to a personal history of breast cancer alone does not justify a recommendation for screening with MR after breast conservation therapy [27]. One retrospective study supports the use of breast MR imaging in this population, demonstrating detection of malignancy in $12 \%$ of women screened with a primary risk factor of a personal history of breast cancer [22]. A separate study by Berg, et al demonstrated that supplemental MRI was less likely to prompt unnecessary recall or biopsy in women with a personal history of breast cancer than in those without a personal history of breast cancer [26]. The choice of adjunct surveillance with Breast MRI is still under investigation and further studies for optimal patient selection are needed.

\section{Other Imaging Modalities}

The role of ultrasound for surveillance is poorly defined in the literature. A few retrospective studies show ultrasound to have a high sensitivity for the detection of malignant lesions in the breast, axilla, and supraclavicular regions. However, these studies failed to demonstrate significant overall survival benefit or change in therapy in each patient population. Ultrasound currently plays a pivotal role in the multimodality modes of surveillance particularly in symptomatic patients. Supplemental ultrasound for asymptomatic patients with a personal history of breast cancer resulted in a $34 \%$ increase in invasive cancer detection compared with annual mammography. Furthermore, the false negative rate was lower in women with a personal history of breast cancer than in women without [26]. PET/CT offers improved accuracy for detection of recurrence compared with the current standard of practice. However, given the increased expense and radiation dose its role in surveillance is primarily as an adjunct imaging tool. Breast Specific Gamma Imaging has no primary role for surveillance.

\section{Post-Mastectomy Imaging}

A second significant challenge in imaging surveillance is identifying the appropriate algorithm and modality for surveillance in the post-mastectomy population. There are no definitive guidelines for surveillance in patients treated by mastectomy with or without reconstruction. Chest wall recurrence in mastectomy patients is between $5 \%$ and $30 \%$ [28, 29]. Case reports and retrospective reviews demonstrate that local recurrence can be detected by surveillance mammography in women with breast reconstruction following mastectomy, but no clear evidence exists to support or discourage routine imaging surveillance in this particular patient population [28-30]. Currently, surveillance imaging algorithms are institutional dependent.

\section{MEDICAL ONCOLOGY ASPECTS}

Following chemotherapy and radiation therapy, current NCCN guidelines recommend history and physical exam twice yearly for 5 years and yearly mammography with the conclusion that intensive follow up, routine tumor markers, and additional imaging modalities in an asymptomatic patient do not increase overall survival. A 2012 Cochrane review by Rojas et al reviewed multicenter randomized controlled trials comparing different follow up strategies in breast cancer patients with stage I, II, or III disease who were disease free after their treatment. The conclusion was that intensive surveillance and shorter follow up intervals provided no significant survival advantage over ordering additional testing as symptoms arise. An example of intensive follow up included physical exam every 3 months for 2 years, followed by every 3 months for 3 years, chest $x$ ray and bone scan every 6 months, and yearly mammogram which was compared to the same physical exam and mammography schedule without any additional testing. Though recurrences were detected earlier in the intensive follow up group, this did not affect the type of treatment offered, nor did it affect estimated 10-year mortality rates $[31,32]$.

A systematic review of the literature in 2007 questioned whether a clinical exam by a specialist improves survival after primary therapy in comparison with self breast exams and mammography. The conclusion was that patient detection and mammography was associated with improved survival compared to clinical examination by a specialist versus a 
general practitioner. This paper also emphasized that in more recent years, the number of treatable relapses diagnosed by mammography increased, likely as a result of technical improvements and enhanced quality assurance [33, 34]. Further reviewing strategies for the early detection of breast cancer recurrence is beyond the scope of this review, but will be further explored in future manuscripts.

\section{MONITORING TREATMENT RESPONSE IN THE NEOADJUVANT SETTING}

Neoadjuvant chemotherapy is well established for the treatment of breast cancer patients with locally advanced disease and/or axillary metastasis with indications for its use continuing to expand. Advantages of neoadjuvant chemotherapy include the reduction of tumor burden allowing a greater number of patients to undergo breast conservation therapy and the preoperative assessment of tumor response to chemotherapy. Tumor size decrease in the neoadjuvant setting is positively associated with disease-free and overall survival. Thus, breast imaging plays a role in evaluating tumor response and informing clinical decisions in the neoadjuvant setting.

\section{SURGICAL ASPECTS}

Following neoadjuvant chemotherapy +/- immunotherapy, patients undergo surgical removal of their tumors. If they were determined to be node-positive prior to neoadjuvant treatment, current $\mathrm{SoC}$ is to also perform an AxLND. The intent of the surgery is to remove the residual tumor and affected nodes for local control of disease but also to definitively assess for treatment response to the neoadjuvant therapy.

Some patients with a complete clinical and radiographic response will still have residual pathologic disease, and surgery is required to make this determination. Standard pathologic assessment is performed on these specimens. However, a minority of women will achieve a complete pathologic response (pCR) with no residual cancer found on pathologic assessment. These women have been shown to have an excellent prognosis and low recurrence rate [35, 36]. Of course, it would be optimal to make this determination in a less invasive manner especially in terms of the nodes. It has been shown that SLNB is accurate after neoadjuvant therapy; however, it is unknown whether this provides adequate local control of disease and disease-free and overall survival equivalent with AxLND [37]. Hopefully, future studies will prove whether full AxLND are required in patients with node-positive disease prior to neoadjuvant therapy if they achieve a complete clinical and radiographic response.
The real dilemma is what more to do for women who do not achieve a pCR. For those patients with good clinical responses but not $\mathrm{pCR}$, it would seem that some adjuvant therapy with low toxicity would be in order such as hormonal or even possibly immunotherapy. However, the larger concern is for those women with minimal clinical and pathologic response. These women are at an extremely high risk for disease recurrence. Many groups are focused on designing and implementing clinical trials to determine the best treatment options for these women who do not achieve $p C R$ after neoadjuvant therapy.

\section{RADIOLOGY ASPECTS}

Contrast enhanced magnetic resonance imaging is the imaging modality of choice for evaluating the initial extent of disease [26, 38]. While breast MR is accurate for evaluation of residual breast cancer after treatment, several studies demonstrate both false-positive and false-negative incidents [39, 40]. Therefore, even in the absence of residual disease on breast MR, definitive surgical resection is required to document $\mathrm{pCR}$ to therapy. Nevertheless, breast $M R$ correlates more accurately with pathologic specimen size when compared to clinical exam, ultrasound, and mammography. Information about residual tumor burden provided by breast $M R$ assists with preoperative planning and guiding surgical management $[38,41]$.

\section{Quantifying Response}

Determining an accurate and repeatable means for evaluating response to therapy remains a challenge. An objective assessment of response of the primary tumor and any metastatic lesions is necessary to measure therapeutic effect. One such method of assessment evaluates the regression in the size of tumors as a measured endpoint, particularly in clinical trials of new chemotherapy agents. In 2000, the Response Evaluation Criteria in Solid Tumours (RECIST) Working Group defined objective criteria to assess for tumor reduction in response to therapy in clinical trials and then later updated these criteria to version 1.1 to include pathologically enlarged lymph nodes and (18)F-fluorodeoxyglucose positron emission tomography (18FDG-PET) data [42, 43]. In the RECIST criteria, up to five measureable lesions $(\geq 10$ $\mathrm{mm}$ in greatest dimension, maximum two per organ, or lymph nodes $\geq 15 \mathrm{~mm}$ in short axis) are identified and the longest diameters are summed and trended to assess for objective tumor response to therapy. Based on these criteria, the RECIST Working Group assigned four categories of response: complete response $(\mathrm{CR})$, partial response (PR), progressive (PD) and stable disease (SD). These criteria are summarized in Table 1. 
Table I: Response Evaluation Criteria in Solid Tumors - RESIST I.I

\begin{tabular}{|l|l|}
\hline Response Classification & Assessment Criteria \\
\hline CR (Complete Response) & $\begin{array}{l}\text { - Disappearance of all target lesions } \\
\text { - Short axis reduction to }<10 \mathrm{~mm} \text { of any pathological lymph nodes }\end{array}$ \\
\hline PR (Partial Response) & $\geq 30 \%$ decrease in sum of target lesion diameters from baseline sum diameters \\
\hline PD (Progressive Disease & $\begin{array}{l}\geq 20 \% \text { increase in sum of target lesion diameters from smallest sum diameter AND } \\
\geq 5 \mathrm{~mm} \text { increase in sum of target lesion diameters }\end{array}$ \\
& $\begin{array}{l}\text { New lesions (one or more) } \\
\text { Unequivocal progression of nontarget lesions }\end{array}$ \\
\hline SD (Stable Disease) & Neither PR nor PD reference the smallest sum diameter \\
\hline
\end{tabular}

Table Modified from Eisenhauer et al [43]

Abnormal foci of increased 18FDG-PET, under RECIST 1.1, must be confirmed by a follow up CT or with the diagnostic quality CT portion (IV and enteric contrast) of a 18FDG PET-CT for inclusion as a measureable lesion.

RECIST criteria are generally used in the setting of clinical trial evaluation of therapy and have not been universally accepted into clinical practice. Advanced imaging modalities such as 18FDG PET or 18FDG PET-CT are described as optional modalities in the NCCN Breast Cancer guidelines [16]. Still, the use of advanced imaging in the assessment of breast cancer response and staging is being utilized. In a recent publication of the patterns of breast cancer imaging in Washington State noted 468 patients out of 9,196 patient included in the study received both breast MRI and CT, or PET or PET-CT [44].

In the setting of neoadjuvant therapy, 18FDG PET has good sensitivity but reduced specificity. In their meta-analysis of the current literature to determine the diagnostic performance of 18FDG PET and PET-CT, Cheng et al., demonstrated that 18FDG PET and PET-CT have a reasonable sensitivity $(0.847,95 \%$ CI 0.793-0.892) in evaluating response to neoadjuvant chemotherapy in breast cancer; however specificity was relativity low $(0.661,95 \%$ CI $0.598-0.720)$. They concluded, "No single diagnostic technique is able to reliably asses pathological response to neoadjuvant chemotherapy, but the combination of other imaging methods (MR, US, mammography) with FDG PET-CT or PET is recommended" [45].

The primary challenges in the neoadjuvant setting include the accurate assessment of early response to therapy and discovering a non-invasive means of accurately predicting pathologic complete response to therapy. Both of these areas are under active investigation and will be discussed in the accompanying manuscript addressing future directions.

\section{MEDICAL ONCOLOGY ASPECTS}

Aside from clinical examination and imaging, tumor markers have also been investigated to predict and monitor response to neoadjuvant chemotherapy. A 2010 retrospective review of patients who received neoadjuvant chemotherapy measured expression of tissue tumor markers found that patients who were hormone receptor (HR) negative (both ER and PgR negative) achieved significantly higher rates of $\mathrm{pCR}$ compared to hormone receptor positive patients $(26 \%$ versus $4 \%$ ). However, only $13 \%$ of the study population achieved a pCR [46]. While these results are intriguing the retrospective nature of the study along with a questionable criteria used for hormone receptor positivity limits the study's applicability.

Serum tumor markers such as CA 15-3 have also been investigated in the neoadjuvant setting. In a prospective study by Al-azawi et al, an elevated CA 15-3 level prior to neo-adjuvant chemotherapy was found to be predictive of a poor pathologic response in only $37.7 \%$ of patients. In addition, when combined with lymphovascular invasion and HER2 overexpression the continued elevation of CA 15-3 after primary therapy for locally advanced breast cancer predicted a worsened disease free survival [47]. This study was limited due to its small size and the fact that less than $35 \%$ of patients received taxane-based chemotherapy. While the results are intriguing, larger prospective trials are needed to help determine whether overall survival may be impacted by tumor marker surveillance in this setting.

The primary role of neoadjuvant therapy is to downstage patients prior to surgery. Presumably, if a patient is not responding to neoadjuvant treatment in early stage breast cancer, then therapy should be halted to pursue definitive therapy. Unfortunately, we are unable to accurately ascertain which patients will respond or not respond to treatment. The current testing for ER, PgR, and HER2 do not reliably predict response to therapy, nor can they be repeatedly measured to monitor response. In addition, as described above, no serum tumor marker has proved reliable in predicting or measuring response.

\section{MONITORING DISEASE RESPONSE IN THE METASTATIC SETTING \\ SURGICAL ASPECTS}

As there are very limited indications for surgery in the metastatic setting, there are no specific monitoring strategies that are surgery specific. Monitoring 
disease after surgical interventions such as metastectomy or palliative debulking. Recommended monitoring strategies are outlined below.

\section{RADIOLOGY ASPECTS}

NCCN guidelines suggest the following for staging evaluation of women with recurrent or metastatic breast cancer: diagnostic chest $\mathrm{CT}$, bone scan, and radiographs of painful long bones or those with abnormal appearance. CT of the abdomen with or without the pelvis may also be considered for restaging. PET/CT is considered an optional modality in this setting and should be considered in situations where standard imaging results are equivocal or suspicious. Per the NCCN guidelines either a bone scan or sodium fluoride PET/CT is recommended to evaluate for bony metastasis unless prior FDG PET/CT already indicated bony metastasis [16].

\section{$\mathrm{PET} / \mathrm{CT}$}

Though PET/CT is considered optional, recent literature suggests that PET/CT is appropriate for restaging of breast cancer patients with documented or suspected recurrent breast cancer. It accurately detects abnormal extra-axillary lymph nodes, detects distant metastases, and often demonstrates recurrent and/or distant disease prior to conventional imaging modalities [48]. Evaluation of breast cancer patients with 18FDG PET or 18FDG PET-CT allows for survey of the chest, abdomen and bones in a single examination with both anatomic and metabolic information useful in the staging, restaging and assessing for therapeutic response. In the setting of asymptomatic patients with rising tumor markers the use of PET/CT may result in early detection of disease and a significant change in management.

Grassetto, et al. looked at 89 patients with breast cancer who had rising serum tumor markers but negative conventional imaging (mammography, ultrasound, contrast enhanced CT, and bone scan). 18FDG PET/CT identified tumor deposits in 40 of the 89 patients leading to change in treatment strategy and improved outcomes [49]. In a recent prospective study involving locally advanced or inflammatory breast cancer patients, 18FDG PET-CT outperformed conventional imaging for detection of bone and liver metastasis as well as distant lymph node involvement leading to a change in clinical stage in 61 of 117 patients (52\%). 18FDG PET-CT had an increased accuracy ( 98.3 versus $89.7 \%$ ) in comparison to planar bone scintigraphy and detected all sites of known liver metastasis in comparison to conventional imaging with CT or ultrasound. Furthermore, when recurrence is detected, PET/CT is useful for determining if there is isolated or distant recurrence. For example, additional lesions not apparent on conventional imaging may be depicted by PET/CT up to $45 \%$ of the time with resultant change in management [48].

Future directions and emerging imaging modalities to monitor disease in the metastatic setting will be described in the paper to follow.

\section{MEDICAL ONCOLOGY ASPECTS}

Monitoring treatment responses for women with metastatic breast cancer can be difficult. With the goals of therapy focused on improving quality of life and overall survival, the challenge has been finding a test that is safe, non-invasive and reliable to assess response. Table 2 summarizes the current guidelines for monitoring disease status in women treated for metastatic breast cancer. Of all the standard monitoring modalities, radiographic examination has been the most readily used to measure treatment response, but some studies report up to $10-40 \%$ of women who have disease that is not measurable [50]. This includes irradiated lesions, pleural effusions, bone only metastatic disease and other difficult to assess metastatic sites. This has led to the investigation of several circulating and tissue-based tumor markers. If elevated at the time of treatment initiation, tumor markers could be helpful for monitoring treatment response. In addition, use of tumor markers have the potential to decrease the use of radiology re-staging studies [51].

Table 2: Current Guidelines for Monitoring Response to Therapy in Breast Cancer Patients

\begin{tabular}{|l|l|l|l|l|}
\hline & $\begin{array}{l}\text { Baseline prior to } \\
\text { new therapy }\end{array}$ & Chemotherapy & Endocrine Therapy & $\begin{array}{l}\text { Restaging if concern for } \\
\text { progression }\end{array}$ \\
\hline Symptom Assessment & Yes & Prior to each cycle & Every 2-3 months & Yes \\
\hline Physical Exam & Yes & Prior to each cycle & Every 2-3 months & Yes \\
\hline Performance Status & Yes & Prior to each cycle & Every 2-3 months & Yes \\
\hline Weight & Yes & Prior to each cycle & Every 2-3 months & Yes \\
\hline LFTs, CBC & Yes & Prior to each cycle & Every 2-3 months & Yes \\
\hline CT scan chest/abd/pelvis & Yes & Every 2-4 cycles & Every 2-6 months & Yes \\
\hline Bone Scan & Yes & Every 2-4 cycles & Every 4-6 months & Yes \\
\hline PET/CT & Optional & Unknown & Unknown & Optional \\
\hline
\end{tabular}

*Adapted from NCCN Guidelines 3.2013 


\section{Circulating Tumor Markers}

ASCO published an update regarding the use of tumor markers in breast cancer in 2007 [15]. This update focused on recommendations for the use of tumor markers for diagnosis, staging, screening, prognosis, predictive value, and monitoring of disease. We will focus on their recommendations regarding the clinical utility of the CA 15-3, CA 27.29 and CEA for monitoring patients with metastatic disease during active therapy. The basic premise is that rising tumor markers are concerning for tumor progression, especially when other clinical finding or imaging are inconclusive.

\section{CA I5-3 and CA 27.29}

Epithelial cells are protected by a mucous barrier consisting of both secreted and transmembrane mucins. MUC- 1 is a transmembrane mucin which can become overexpressed in epithelial cancer cells. Overexpression can lead to loss of epithelial polarity which in turn can cause activation of tyrosine kinases leading to downstream signaling and cancer cell survival [52]. Several murine antibodies to MUC-1 gene products are available including CA $15-3$ and CA 27.29 which detect soluble forms of MUC-1 [53] . CA 15-3 is an FDA approved assay that detects shed or soluble forms of MUC-1 protein [54]. Currently, the most common assay for CA 15-3 detects antigens reacting with 2 monoclonal antibodies, DF3 and 115-D8 [55]. CA 27.29 also detects MUC-1 through the monoclonal antibody BR 27.29 , specific to an 8 amino acid tandem repeat region which partially overlaps with the binding site of DF3 tested for with the CA 15-3 assay. It was shown to be effective for the early detection of recurrence in the follow up of previously disease free patients [56]. While some studies have suggested that CA 27.29 is a more sensitive test [57]. A more recent study suggests that test value differences may have more to do with assay calibration rather than differences in specificity of the assays [58]. The majority of clinical data regarding the utility of MUC-1 based tumor markers for monitoring metastatic breast cancer has been done using CA 15-3 assays.

Early retrospective studies confirmed that CA 15-3 was a sensitive tumor marker, present in over $70 \%$ of women with metastatic breast cancer $[59,60]$. Furthermore, when a bideterminant immunoassay for CA 15-3, with monoclonal antibodies to DF3 and 115 -D8, was used it was elevated in $73 \%$ of metastatic breast cancer patients versus only 55\% CEA elevation in the same patient population. Todini et al. showed that use of CA $15-3$ was $60.3 \%$ sensitive and $71.1 \%$ specific when monitoring response to therapy [61]. This retrospective study used an increase in CA 15-3 $\geq$
$25 \%$ from initial levels to correlate with progressive disease (PD), while decreases in tumor markers $\geq 25 \%$ from baseline seemed correlated with disease response. Finally, a change in CA $15-3$ of $\leq 25 \%$ correlated with stable disease (SD). CA 15-3 correlated with disease progression, regression, or stability in more patients than CEA $(60.3 \%$ vs. $39.6 \%$; $p=0.02)$ Use of both CEA and CA $15-3$ increased specificity to $95.5 \%$.

\section{CEA (carcinoembryonic antigen)}

Current guidelines recommend monitoring the serum tumor marker carcinoembryonic antigen (CEA) in metastatic breast cancer to help guide treatment decisions, in conjunction with standard clinical monitoring and imaging, if initial values are elevated at the diagnosis of disseminated disease [15]. Early studies of CEA monitoring in the metastatic setting showed that half of patients had an elevated CEA prior to initiation of chemotherapy [62]. In those with clinical response, a decrease in CEA was found in $94 \%$ of cases and in those patients who had normalization of CEA levels, their duration of response was close to 22 months vs. 9 months in those whose CEA levels did not normalize. Although only $40-50 \%$ of patients with metastatic breast cancer have elevated CEA levels, adding the measurement of CEA to other MUC-1 antigen testing (CA15-3) may result in a slightly improved sensitivity [63].

Several studies have described situations where the CEA is elevated, while the MUC-1 associated tumor markers, CA15.3 and CA 27.29 are normal [64-66]. One study found that $35 \%$ of patients with normal CA15-3 levels may have elevations of other tumor markers (CEA and/or CA 125) [67]. This is in keeping with current recommendations, which call for evaluating a CEA level in conjunction with one of the MUC-1 assays on the initial presentation of metastatic breast cancer. Ongoing CEA monitoring is only advised if the MUC-1 studies are in the normal range. However, current guidelines do not address monitoring of CA-125, a tumor marker commonly used in monitoring epithelial ovarian cancer. Available data suggest CA-125 levels are elevated in up to $84 \%$ metastatic breast cancer patients, so monitoring of this tumor marker maybe part of future guidelines [68, 69], as adding CA- 125 to standard tumor marker assays, may increase the sensitivity to as high as $90 \%$ in the metastatic setting [68].

\section{Prospective studies of tumor markers}

To further define the role of tumor markers in the monitoring of metastatic disease, several prospective studies were performed. Clinical utility of individual tumor markers was prospectively evaluated using CEA, CA 15-3 and TPS (tissue polypeptide spe- 
cific antigen)[70]. One hundred twenty-nine women with metastatic breast cancer were followed for 6 months. The sensitivity for CA $15-3$ was $73 \%$ vs. $69 \%$ for TPS. In another small, multi-center prospective trial, 83 women with metastatic breast cancer were assessable for following an established biochemical index, which included measurement of CA 15-3, CEA and ESR [71]. Treatment response was measured by standardized criteria at baseline and at 3 month intervals. Eighty four percent of women had an elevation of at least one biochemical marker upon initial assessment. In this study, changes in tumor markers corresponded to standard criteria for disease progression at first assessment in 34/37 patients. In the 3 women whose markers were falling at 3 months, they were found to have progressive disease by standardized criteria. In women with late progression (defined as $>6$ months from initiation of treatment) 13/17 (67\%) had tumor marker elevation between 3-9 months prior to progression as measured by standard criteria. Three women that never had an elevated index remained in remission for the duration of this study.

In 2004, a prospective Japanese study analyzed 108 women with metastatic breast cancer who had sequential tumor marker (CEA, CA 15-3, and NCC-ST-439) measurements drawn every 4 weeks during treatment [72]. This study concluded that changes in tumor markers correlated to response to therapy. In addition, if a $\geq 20 \%$ decrease in tumor marker was noted, this predicted a longer time to progression. One of the more interesting findings was that patients followed with CA 15-3 who had confirmed response to therapy had an initial rise $(\geq 20 \%)$ in their tumor marker measurement $21 \%$ of the time. The authors cautioned regarding a possible "spike" phenomenon shortly after the initiation of therapy which has been seen in other studies [73, 74].

Finally, the largest prospective study looked at the prognostic significance of CA 15-3 levels during anthracycline-based chemotherapy for metastatic breast cancer patients [75]. In this study, 526 patients had serum CA 15-3 measurements obtained at baseline, 3 and 6 months after initiation of chemotherapy while prospectively enrolled in five phase II-III trials. Median time to progression was 15.3 months in women with normal tumor markers throughout the study time, 11.7 for those with a $\geq 25 \%$ decrease, 9.6 months for those with elevated CA 15-3 that did not change significantly, and 8.6 months for those with increased tumor markers $(p<0.001)$. The authors suggested that for women with negative CA 15-3 at presentation, continued monitoring was warranted due to the significant worsening in prognosis for these women who become positive. In this study, woman who did not have an elevation at baseline and remained normal had an overall survival of 42.3 months. Despite the prognostic value of the CA 15-3, up to $23.7 \%$ of patients had a false positive change in CA 15-3 levels and a false negative rate up to $46.2 \%$ leading to specificity at 6 months of only $53.8 \%$.

\section{Current Recommendations}

Based on this data, ASCO has recommended that the MUC-1 assays, CA 15-3 and CA 27.29, can be used for monitoring patients with metastatic breast cancer during active therapy (See Table 3). These assays should be used in conjunction with diagnostic imaging, history and physical examination. Currently, they do not recommend use of CA 15-3 or CA 27.29 alone for monitoring disease but recognize that in the absence of measurable disease an increase may indicate disease progression. They also recommend caution interpreting a rising MUC-1 associated assays during the first 4-6 weeks of treatment as spurious rises can occur.

Table 3: Current standards for using tumor markers in the metastatic setting

\begin{tabular}{|l|l|}
\hline $\begin{array}{l}\text { CA 15-3 } \\
\text { CA 27.29 }\end{array}$ & $\begin{array}{l}\text { NCCN: Optional } \\
\text { ASCO: For monitoring patients with metastatic disease } \\
\text { during therapy in combination with diagnostic imag- } \\
\text { ing, history, and exam. }\end{array}$ \\
\hline CEA & $\begin{array}{l}\text { NCCN: Optional } \\
\text { ASCO: For monitoring patients with metastatic disease } \\
\text { during active therapy in combination with diagnostic } \\
\text { imaging, history, and exam. }\end{array}$ \\
& \multicolumn{2}{|c|}{ Adapted from NCCN Guidelines 3.2013 and ASCO Tumor Marker Guidelines }
\end{tabular}
(2007).

Present data are insufficient to recommend use of any of these three tumor markers ALONE for monitoring response to treatment, although in the absence of measurable disease, increasing levels may point to progressive disease. Caution is recommended when interpreting results as rising levels during the first 4-6 weeks after new treatment is initiated may not be clinically significant.

\section{CONCLUSION}

Given the prevalence of breast cancer patients in the United States, future studies aimed at improving the monitoring of disease are paramount. We have described the common clinical scenarios and standard of care for breast cancer patients undergoing surveillance and monitoring of treatment responses. In the adjuvant setting, the challenge has been improving early detection and more importantly, how to translate early detection into increased survival.

Determining which patients are at higher risk of relapse may aid in this challenge. In order to help identify high risk patients there has been a national 
shift towards treating more women with neo-adjuvant therapy. With the use of new microarray and DNA based assays, the ability to obtain tissue to assess for predictive and prognostic markers before and after neo-adjuvant therapy may help us determine which woman may benefit from enhanced surveillance.

Finally, the challenge in the fight against metastatic disease is to provide safe, inexpensive and accurate tumor markers assessment for our patients. Only through well-designed and standardized clinical trials will we be able to move the field forward. More research in the metastatic setting may also help us discover new novel agents, markers or techniques that might be able to be pushed forward into the adjuvant or even average-risk screening population in order to find new ways to improve breast cancer-specific mortality.

\section{Competing Interests}

The authors have declared that no competing interest exists.

\section{References}

1. Siegel R, DeSantis C, Virgo K, Stein K, Mariotto A, Smith T, et al. Cancer treatment and survivorship statistics, 2012. CA Cancer J Clin. 2012; 62: 220-41. doi:10.3322/caac.21149.

2. Berry DA, Cronin KA, Plevritis SK, Fryback DG, Clarke L, Zelen M, et al. Effect of screening and adjuvant therapy on mortality from breast cancer. $\mathrm{N}$ Engl J Med. 2005; 353: 1784-92. doi:10.1056/NEJMoa050518.

3. Davies C, Pan H, Godwin J, Gray R, Arriagada R, Raina V, et al. Long-term effects of continuing adjuvant tamoxifen to 10 years versus stopping at 5 years after diagnosis of oestrogen receptor-positive breast cancer: ATLAS, a randomised trial. Lancet. 2012. doi:10.1016/S0140-6736(12)61963-1.

4. Piccart-Gebhart MJ, Burzykowski T, Buyse M, Sledge G, Carmichael J, Luck $\mathrm{HJ}$, et al. Taxanes alone or in combination with anthracyclines as first-line therapy of patients with metastatic breast cancer. J Clin Oncol. 2008; 26: 1980-6. doi:10.1200/JCO.2007.10.8399.

5. Ploos van Amstel FK, van den Berg SW, van Laarhoven HW, Gielissen MF, Prins JB, Ottevanger PB. Distress screening remains important during follow-up after primary breast cancer treatment. Support Care Cancer. 2013. doi:10.1007/s00520-013-1764-0.

6. Singletary SE. Surgical margins in patients with early-stage breast cancer treated with breast conservation therapy. Am J Surg. 2002; 184: 383-93. doi:S0002961002010127 [pii].

7. Giuliano AE, Hunt KK, Ballman KV, Beitsch PD, Whitworth PW, Blumencranz $\mathrm{PW}$, et al. Axillary dissection vs no axillary dissection in women with invasive breast cancer and sentinel node metastasis: a randomized clinical trial. JAMA. 2011; 305: 569-75. doi:10.1001/jama.2011.90.

8. Clarke M, Collins R, Darby S, Davies C, Elphinstone P, Evans E, et al. Effects of radiotherapy and of differences in the extent of surgery for early breast cancer on local recurrence and 15-year survival: an overview of the randomised trials. Lancet. 2005; 366: 2087-106. doi:10.1016/S0140-6736(05)67887-7.

9. Houssami N, Ciatto S. Mammographic surveillance in women with a personal history of breast cancer: how accurate? How effective? Breast. 2010; 19: 439-45. doi:10.1016/j.breast.2010.05.010.

10. Lu WL, Jansen L, Post WJ, Bonnema J, Van de Velde JC, De Bock GH. Impact on survival of early detection of isolated breast recurrences after the primary treatment for breast cancer: a meta-analysis. Breast Cancer Res Treat. 2009; 114: 403-12. doi:10.1007/s10549-008-0023-4.

11. Orel SG, Fowble BL, Solin LJ, Schultz DJ, Conant EF, Troupin RH. Breast cancer recurrence after lumpectomy and radiation therapy for early-stage disease: prognostic significance of detection method. Radiology. 1993; 188: 189-94.

12. Arasu VA, Joe BN, Lvoff NM, Leung JW, Brenner RJ, Flowers CI, et al. Benefit of semiannual ipsilateral mammographic surveillance following breast conservation therapy. Radiology. 2012; 264: 371-7. doi:10.1148/radiol.12111458.

13. Carlson RW, Allred DC, Anderson BO, Burstein HJ, Carter WB, Edge SB, et al. Breast Cancer. Journal of the National Comprehensive Cancer Network. 2009; 7: $122-92$.
14. Khatcheressian J, Hurley P, Bentug E, Esserman LJ, Grunfeld E, Halberg F. Breast Cancer Follow-Up and Management After Primary Treatment: American Society of Clinical Oncology Clinical Practice Guideline Update. Journal of Clinical Oncology. 2013; 31: 961-5.

15. Harris L, Fritsche H, Mennel R, Norton L, Ravdin P, Taube S, et al. American Society of Clinical Oncology 2007 update of recommendations for the use of tumor markers in breast cancer. J Clin Oncol. 2007; 25: 5287-312. doi:10.1200/JCO.2007.14.2364.

16. NCCN. NCCN Clinical Practice Guidelines in Oncology (NCCN Guidelines): breast cancer-version 3.2013. 2013.

17. Gunia SR, Merrigan TL, Poulton TB, Mamounas EP. Evaluation of appropriate short-term mammographic surveillance in patients who undergo breast-conserving Surgery (BCS). Ann Surg Oncol. 2012; 19: 3139-43. doi:10.1245/s10434-012-2578-x

18. Lin K, Eradat J, Mehta NH, Bent C, Lee SP, Apple SK, et al. Is a short-interval postradiation mammogram necessary after conservative surgery and radiation in breast cancer? Int J Radiat Oncol Biol Phys. 2008; 72: 1041-7. doi:10.1016/j.ijrobp.2008.02.017.

19. McNaul D, Darke M, Garg M, Dale P. An evaluation of post-lumpectomy recurrence rates: is follow-up every 6 months for 2 years needed? J Surg Oncol. 2013; 107: 597-601. doi:10.1002/jso.23298.

20. Lee CH, Dershaw DD, Kopans D, Evans P, Monsees B, Monticciolo D, et al. Breast Cancer Screening With Imaging: Recommendations From the Society of Breast Imaging and the ACR on the Use of Mammography, Breast MRI, Breast Ultrasound, and Other Technologies for the Detection of Clinically Occult Breast Cancer. Journal of the American College of Radiology. 2010; 7: 18-27. doi:10.1016/j.jacr.2009.09.022.

21. Dershaw DD. Mammography in patients with breast cancer treated by breast conservation (lumpectomy with or without radiation). AJR Am J Roentgenol. 1995; 164: 309-16. doi:10.2214/ajr.164.2.7839960.

22. Brennan S, Liberman L, Dershaw DD, Morris E. Breast MRI screening of women with a personal history of breast cancer. AJR Am J Roentgenol. 2010; 195: 510-6. doi:10.2214/AJR.09.3573.

23. Lehman CD, Gatsonis C, Kuhl CK, Hendrick RE, Pisano ED, Hanna L, et al. MRI evaluation of the contralateral breast in women with recently diagnosed breast cancer. N Engl J Med. 2007; 356: 1295-303. doi:10.1056/NEJMoa065447.

24. Liberman L, Morris EA, Dershaw DD, Abramson AF, Tan LK. MR imaging of the ipsilateral breast in women with percutaneously proven breast cancer. AJR Am J Roentgenol. 2003; 180: 901-10. doi:10.2214/ajr.180.4.1800901.

25. Quinn EM, Coveney AP, Redmond HP. Use of magnetic resonance imaging in detection of breast cancer recurrence: a systematic review. Ann Surg Oncol. 2012; 19: 3035-41. doi:10.1245/s10434-012-2341-3.

26. Berg WA, Zhang Z, Lehrer D, Jong RA, Pisano ED, Barr RG, et al. Detection of breast cancer with addition of annual screening ultrasound or a single screening MRI to mammography in women with elevated breast cancer risk. JAMA. 2012; 307: 1394-404. doi:10.1001/jama.2012.388.

27. Saslow D, Boetes C, Burke W, Harms S, Leach MO, Lehman CD, et al. American Cancer Society guidelines for breast screening with MRI as an adjunct to mammography. CA Cancer J Clin. 2007; 57: 75-89. doi:57/2/75 [pii].

28. Barnsley GP, Grunfeld E, Coyle D, Paszat L. Surveillance mammography following the treatment of primary breast cancer with breast reconstruction: a systematic review. Plast Reconstr Surg. 2007; 120: 1125-32. doi:10.1097/01.prs.0000279143.66781.9a.

29. Destounis S, Morgan R, Arieno A, Seifert P, Somerville P, Murphy P. A review of breast imaging following mastectomy with or without reconstruction in an outpatient community center. Breast Cancer. 2011; 18: 259-67. doi:10.1007/s12282-011-0287-0.

30. Helvie MA, Bailey JE, Roubidoux MA, Pass HA, Chang AE, Pierce LJ, et al. Mammographic screening of TRAM flap breast reconstructions for detection of nonpalpable recurrent cancer. Radiology. 2002; 224: 211-6.

31. Rosselli Del Turco M, Palli D, Cariddi A, Ciatto S, Pacini P, Distante V. Intensive diagnostic follow-up after treatment of primary breast cancer. A randomized trial. National Research Council Project on Breast Cancer follow-up. JAMA. 1994; 271: 1593-7.

32. Palli D, Russo A, Saieva C, Ciatto S, Rosselli Del Turco M, Distante V, et al. Intensive vs clinical follow-up after treatment of primary breast cancer: 10-year update of a randomized trial. National Research Council Project on Breast Cancer Follow-up. JAMA. 1999; 281: 1586. doi:jlt0505 [pii].

33. Montgomery D, Krupa K, Cooke T. Follow up in breast cancer: does routine clinical examination improve outcome? A systematic review of the literature. British Journal of Cancer. 2007; 97: 10.

34. Grunfeld E, Fitzpatrick R, Mant D, Yudkin P, Adewuyi-Dalton R, Stewart J, et al. Comparison of breast cancer patient satisfaction with follow-up in primary care versus specialist care: results from a randomized controlled trial. Br J Gen Pract. 1999; 49: 705-10.

35. Fisher B, Bryant J, Wolmark N, Mamounas E, Brown A, Fisher ER, et al. Effect of preoperative chemotherapy on the outcome of women with operable breast cancer. Journal of Clinical Oncology. 1998; 16: 2672-85.

36. Redden MH, Fuhrman GM. Neoadjuvant chemotherapy in the treatment of breast cancer. Surg Clin North Am. 2013; 93: 493-9. doi:10.1016/j.suc.2013.01.006.

37. Hunt KK, Yi M, Mittendorf EA, Guerrero C, Babiera GV, Bedrosian I, et al. Sentinel lymph node surgery after neoadjuvant chemotherapy is accurate and reduces the need for axillary dissection in breast cancer patients. Ann Surg. 2009; 250: 558-66. doi:10.1097/SLA.0b013e3181b8fd5e. 
38. Argus A. Clinical Indications for breast MR. Applied Radiology. 2010; 39: 10-9.

39. Marinovich ML, Houssami N, Macaskill P, Sardanelli F, Irwig L, Mamounas $\mathrm{EP}$, et al. Meta-analysis of magnetic resonance imaging in detecting residual breast cancer after neoadjuvant therapy. J Natl Cancer Inst. 2013; 105: 321-33. doi:10.1093/jnci/djs528.

40. Woodhams R, Kakita S, Hata H, Iwabuchi K, Kuranami M, Gautam S, et al. Identification of residual breast carcinoma following neoadjuvant chemotherapy: diffusion-weighted imaging--comparison with contrast-enhanced MR imaging and pathologic findings. Radiology. 2010; 254: 357-66. doi:10.1148/radiol.2542090405.

41. Croshaw R, Shapiro-Wright H, Svensson E, Erb K, Julian T. Accuracy of clinical examination, digital mammogram, ultrasound, and MRI in determining postneoadjuvant pathologic tumor response in operable breast cancer patients. Ann Surg Oncol. 2011; 18: 3160-3. doi:10.1245/s10434-011-1919-5.

42. Therasse P, Arbuck SG, Eisenhauer EA, Wanders J, Kaplan RS, Rubinstein L, et al. New guidelines to evaluate the response to treatment in solid tumors. European Organization for Research and Treatment of Cancer, National Cancer Institute of the United States, National Cancer Institute of Canada. J Natl Cancer Inst. 2000; 92: 205-16.

43. Eisenhauer EA, Therasse P, Bogaerts J, Schwartz LH, Sargent D, Ford R, et al. New response evaluation criteria in solid tumours: revised RECIST guideline (version 1.1). Eur J Cancer. 2009; 45: 228-47. doi:10.1016/j.ejca.2008.10.026.

44. Gold LS, Buist DSM, Loggers ET, Etzioni R, Kessler L, Ramsey SD, et al. Advanced Diagnostic Breast Cancer Imaging: Variation and Patterns of Care in Washington State. Journal of Oncology Practice. 2013. doi:10.1200/jop.2012.000796.

45. Cheng X, Li Y, Liu B, Xu Z, Bao L, Wang J. 18F-FDG PET/CT and PET for evaluation of pathological response to neoadjuvant chemotherapy in breast cancer: a meta-analysis. Acta Radiol. 2012; 53: 615-27. doi:10.1258/ar.2012.110603.

46. Precht LM, Lowe K, Atwood M, Beatty D. Neoadjuvant Chemotherapy of Breast Cancer: Tumor Markers as Predictors of Pathologic Response, Recurrence, and Survival. The Breast Journal. 2010; 16: 8 .

47. Al-azawi D, Kelly G, Myers E, McDermott EW, Hill AD, Duffy MJ, et al. CA $15-3$ is predictive of response and disease recurrence following treatment in locally advanced breast cancer. BMC Cancer. 2006;6:220.

48. Groheux D, Espié M, Giacchetti S, Hindié E. Performance of FDG PET/CT in the Clinical Management of Breast Cancer. Radiology. 2013; 266: 388-405. doi:10.1148/radiol.12110853.

49. Grassetto G, Fornasiero A, Otello D, Bonciarelli G, Rossi E, Nashimben O, et al 18F-FDG-PET/CT in patients with breast cancer and rising Ca 15-3 with negative conventional imaging: a multicentre study. Eur J Radiol. 2011; 80: 828-33. doi:10.1016/j.ejrad.2010.04.029.

50. Cheung KL, Graves CR, Robertson JF. Tumour marker measurements in the diagnosis and monitoring of breast cancer. Cancer Treat Rev. 2000; 26: 91-102. doi:10.1053/ctrv.1999.0151.

51. Wu LM, Hu J, Gu HY, Hua J, Xu JR Can diffusion-weighted magnetic resonance imaging (DW-MRI) alone be used as a reliable sequence for the preoperative detection and characterisation of hepatic metastases? A meta-analysis. Eur J Cancer. 2013; 49: 572-84. doi:10.1016/j.ejca.2012.08.021.

52. Kufe DW. Mucins in cancer: function, prognosis, and therapy. Nat Rev Cancer. 2009; 9: 874-85.

53. Taylor-Papadimitriou J. Report on the first international workshop on carcinoma-associated mucins. Int J Cancer. 1991; 49: 1-5.

54. Hayes DF, Sekine H, Ohno T, Abe M, Keefe K, Kufe DW. Use of a murine monoclonal antibody for detection of circulating plasma DF3 antigen levels in breast cancer patients. J Clin Invest. 1985; 75: 1671-8. doi:10.1172/JCI111875.

55. Duffy MJ, Evoy D, McDermott EW. CA 15-3: uses and limitation as a biomarker for breast cancer. Clin Chim Acta. 2010; 411: 1869-74. doi:10.1016/j.cca.2010.08.039

56. Chan DW, Beveridge RA, Muss $H$, Fritsche HA, Hortobagyi G, Theriault R, et al. Use of Truquant BR radioimmunoassay for early detection of breast cancer recurrence in patients with stage II and stage III disease. J Clin Oncol. 1997; 15: 2322-8.

57. Gion M, Mione R, Leon AE, Dittadi R. Comparison of the diagnostic accuracy of CA27.29 and CA15.3 in primary breast cancer. Clin Chem. 1999; 45: 630-7.

58. Klee GG, Schreiber WE. MUC1 Gene-Derived Glycoprotein Assays for Monitoring Breast Cancer (CA 15-3, CA 27.29, BR). Arch Pathol Lab Med. 2004; 128: 1131-5.

59. Hayes DF, Zurawski VR, Jr., Kufe DW. Comparison of circulating CA15-3 and carcinoembryonic antigen levels in patients with breast cancer. J Clin Oncol. 1986; 4: 1542-50

60. Kufe D, Inghirami G, Abe M, Hayes D, Justi-Wheeler H, Schlom J. Differential reactivity of a novel monoclonal antibody (DF3) with human malignant versus benign breast tumors. Hybridoma. 1984; 3: 223-32.

61. Tondini C, Hayes DF, Gelman R, Henderson IC, Kufe DW. Comparison of CA15-3 and carcinoembryonic antigen in monitoring the clinical course of patients with metastatic breast cancer. Cancer Res. 1988; 48: 4107-12.

62. Mughal AW, Hortobagyi GN, Fritsche HA, Buzdar AU, Yap HY, Blumenschein GR. Serial plasma carcinoembryonic antigen measurements during treatment of metastatic breast cancer. JAMA. 1983; 249: 1881-6.

63. Guadagni F, Ferroni P, Carlini S, Mariotti S, Spila A, Aloe S, et al. A re-evaluation of carcinoembryonic antigen (CEA) as a serum marker for breast cancer: a prospective longitudinal study. Clin Cancer Res. 2001; 7: 2357-62.
64. Lokich JJ. CEA: a monitor of therapy for breast and colon cancer. Am Fam Physician. 1978; 17: 173-6.

65. Loprinzi CL, Tormey DC, Rasmussen P, Falkson G, Davis TE, Falkson HC, et al. Prospective evaluation of carcinoembryonic antigen levels and alternating chemotherapeutic regimens in metastatic breast cancer. J Clin Oncol. 1986; 4: 46-56.

66. Woo KB, Waalkes TP, Ahmann DL, Tormey DC, Gehrke CW, Oliverio VT. A quantitative approach to determining disease response during therapy using multiple biologic markers: application to carcinoma of the breast. Cancer. 1978; 41: 1685-703.

67. Yerushalmi R, Tyldesley S, Kennecke H, Speers C, Woods R, Knight B, et al. Tumor markers in metastatic breast cancer subtypes: frequency of elevation and correlation with outcome. Ann Oncol. 2012; 23: 338-45. doi:10.1093/annonc/mdr154.

68. Baskic D, Ristic P, Matic S, Bankovic D, Popovic S, Arsenijevic N. Clinical evaluation of the simultaneous determination of CA 15-3, CA 125 and sHER2 in breast cancer. Biomarkers. 2007; 12: 657-67. doi:10.1080/13547500701520563.

69. Berruti A, Tampellini M, Torta M, Buniva T, Gorzegno G, Dogliotti L. Prognostic value in predicting overall survival of two mucinous markers: CA 15-3 and CA 125 in breast cancer patients at first relapse of disease. Eur J Cancer. 1994; 30A: 2082-4.

70. van Dalen A. Significance of cytokeratin markers TPA, TPA (cyk), TPS and CYFRA 21.1 in metastatic disease. Anticancer Res. 1996; 16: 2345-9.

71. Robertson JF, Jaeger W, Syzmendera JJ, Selby C, Coleman R, Howell A, et al. The objective measurement of remission and progression in metastatic breast cancer by use of serum tumour markers. European Group for Serum Tumour Markers in Breast Cancer. Eur J Cancer. 1999; 35: 47-53.

72. Kurebayashi J, Nishimura R, Tanaka K, Kohno N, Kurosumi M, Moriya T, et al. Significance of serum tumor markers in monitoring advanced breast cancer patients treated with systemic therapy: a prospective study. Breast Cancer. 2004; 11: 389-95.

73. Kim HS, Park YH, Park MJ, Chang MH, Jun HJ, Kim KH, et al. Clinical significance of a serum CA15-3 surge and the usefulness of CA15-3 kinetics in monitoring chemotherapy response in patients with metastatic breast cancer. Breast Cancer Res Treat. 2009; 118: 89-97. doi:10.1007/s10549-009-0377-2.

74. Wu SC, Chou FF, Rau KM. Clinical significance of a serum CA 15-3 surge and the usefulness of CA 15-3 kinetics in monitoring chemotherapy response in patients with metastatic breast cancer. Breast Cancer Res Treat. 2010; 124: 879-82. doi:10.1007/s10549-010-1117-3.

75. Tampellini M, Berruti A, Bitossi R, Gorzegno G, Alabiso I, Bottini A, et al. Prognostic significance of changes in CA 15-3 serum levels during chemotherapy in metastatic breast cancer patients. Breast Cancer Res Treat. 2006; 98: 241-8. doi:10.1007/s10549-005-9155-y. 